\title{
Colchicine as an adjunct to heparin for prophylaxis of venous thromboembolism in patients with COVID-19
}

\author{
Chia Siang Kow ${ }^{1}\left[\right.$. Syed Shahzad Hasan ${ }^{2,3}(\mathbb{D}$
}

Received: 6 November 2020 / Accepted: 11 November 2020 / Published online: 2 January 2021

○) Springer-Verlag GmbH Germany, part of Springer Nature 2021

The comprehensive review by Misra et al. [1] on the effects of antirheumatic drugs, including hydroxychloroquine, methotrexate and colchicine, in patients with coronavirus disease 2019 (COVID-19), along with discussion on their positive and negative sides to guide repurposing of these antirheumatic drugs against COVID-19, is commendable. Particularly, we read with interest the authors' viewpoints regarding the potential therapeutic roles of colchicine in patients with COVID-19, and we would like to complement to their discussion on colchicine based on recent understandings on the pathophysiology of COVID-19.

The findings reported by Busch et al. from their study [2] of 228 patients with COVID-19, in which citrullinated histone H3, a marker of neutrophil extracellular traps (NETs) formation, was detected in those with severe disease but not in those with mild disease, had recently raised the interest of medical community. Busch et al. [2] reported that serum samples from patients with severe disease showed NETs that stained positive for DNA and citrullinated H3. Such findings suggested that NETs formation with the release of DNA and histones could trigger the contact pathway of coagulation and subsequent development of thrombosis in patients with COVID-19, especially those with severe disease.

Their findings have been substantiated by another prospective study [3] which demonstrated the pathophysiological role of NETs in patients with COVID-19. The authors discovered the correlation between plasma NET levels and disease severity in patients with COVID-19, in which plasma NET levels in patients with COVID-19 correlated

Chia Siang Kow

chiasiang_93@hotmail.com

1 School of Postgraduate Studies, International Medical University, Kuala Lumpur, Malaysia

2 Department of Pharmacy, University of Huddersfield, Huddersfield, United Kingdom

3 School of Biomedical Sciences \& Pharmacy, University of Newcastle, Callaghan, Australia directly with the Sequential Organ Failure Assessment score. Another important discovery from the study was the interaction of NETs with platelets as shown in the autopsy lung samples of patients with COVID-19, which suggested the involvement of NETs in the development of thrombosis in patients with COVID-19.

Prophylactic-dose anticoagulation with low molecular weight heparins (or unfractionated heparin for patients with renal impairment) has been recommended for venous thromboembolism (VTE) prevention in hospitalized patients with COVID-19, although such intensity of anticoagulation may not be sufficient to prevent development of thrombosis in those with severe disease [4]. While some clincians have advocated the use of therapeutic-dose anticoagulation with low molecular weight heparins for prophylaxis of VTE in this patient population, it is nevertheless accompanied by high risk of bleeding [5]. Bleeding may in turn lead to more complicated illnesss including death among patients with COVID-19, and therefore the use of therapeutic anticoagulation with low molecular weight heparins has an unclear risk-benefit ratio.

Since NETs contribute to immunothrombosis in patients with COVID-19, especially those with severe disease, antiNETs therapeutics may be useful as an adjunct to prophylactic-dose low molecular weight heparins for the prevention of VTE in this patient population. Colchicine has emerged as a potential treatment for patients with COVID-19 as discussed by Misra et al. [1], and interestingly colchicine could suppress NET formation [6]. Colchicine stabilizes the cytoskeleton, thereby attenuating chromatin swelling and subsequent NET release from neutrophils. Of note, a randomized clinical trial [7] reported that patients with COVID-19 who were randomized to colchicine treatment had statistically significantly lower peak median D-dimer concentration, which is a sensitive marker of thrombosis, compared to those who were randomized to control treatment. The same was observed in a case-control study [8] among hospitalized patients with moderate-to-severe course 
of COVID-19, where patients in the colchicine group showed a more significant decrease in the D-dimer level compared to the control group. Therefore, we believe that colchicine (alongside a prophylactic-dose low molecular weight heparin) is certainly worthy of more evaluation in terms of its efficacy for the prophylaxis of VTE, in addition to its efficacy for clinical improvement, among hospitalized patients with COVID-19. Specifically, hospitalized patients with a severe course of COVID-19 and concurrent inflammatory rheumatic diseases may be prioritized for this VTE prophylactic approach with colchicine (alongside a prophylactic-dose low molecular weight heparin), since patients with inflammatory rheumatic diseases have a higher baseline risk of VTE than in the general population [9].

\section{Funding None.}

\section{Compliance with ethical standards}

Conflict of interest Chia Siang Kow and Syed Shahzad Hasan report no conflicts of interest.

\section{References}

1. Misra DP, Gasparyan AY, Zimba O (2020) Benefits and adverse effects of hydroxychloroquine, methotrexate and colchicine: searching for repurposable drug candidates. RheumatolInt 40(11):1741-1751
2. Busch MH, Timmermans S, Nagy M et al (2020) Neutrophils and contact activation of coagulation as potential drivers of COVID19. Circulation 142(18):1787-1790

3. Middleton EA, He XY, Denorme F et al (2020) Neutrophil extracellular traps contribute to immunothrombosis in COVID-19 acute respiratory distress syndrome. Blood 136(10):1169-1179

4. Hasan SS, Radford S, Kow CS, Zaidi STR (2020) Venous thromboembolism in critically ill COVID-19 patients receiving prophylactic or therapeutic anticoagulation: a systematic review and meta-analysis. J Thromb Thrombolysis 50(4):814-821

5. Musoke N, Lo KB, Albano J et al (2020) Anticoagulation and bleeding risk in patients with COVID-19. Thromb Res 196:227230 (published online ahead of print, $2020 \mathrm{Aug} 24$ )

6. Vaidya K, Tucker B, Kurup R, et al. Colchicine inhibits neutrophil extracellular trap formation in acute coronary syndrome patients after percutaneous coronary intervention. Preprint. medRxiv. 2020;2020.04.20.20034025

7. Deftereos SG, Giannopoulos G, Vrachatis DA et al (2020) Effect of colchicine vs standard care on cardiac and inflammatory biomarkers and clinical outcomes in patients hospitalized with coronavirus disease 2019: the GRECCO-19 randomized clinical trial. JAMA Netw Open 3(6):e2013136

8. Sandhu T, Tieng A, Chilimuri S, Franchin G (2020) A case control study to evaluate the impact of colchicine on patients admitted to the hospital with moderate to severe COVID-19 infection. Can J Infect Dis Med Microbiol 2020:8865954

9. Lee JJ, Pope JE (2014) A meta-analysis of the risk of venous thromboembolism in inflammatory rheumatic diseases. Arthritis Res Ther 16(5):435

Publisher's Note Springer Nature remains neutral with regard to jurisdictional claims in published maps and institutional affiliations. 